\title{
Multiple myeloma and AL amyloid cardiomyopathy
}

\author{
Varayini Pankayatselvan, Inbar Raber, Alvin S. Chen, Bharath G. Rathakrishnan, Pablo A. Quintero, Marwa A. Sabe, \\ Jason A. Freed, Arthur R. Garan \\ Beth Israel Deaconess Medical Center, United States
}

Received: May 20, 2020

Accepted: July 9, 2020

Online Published: August 28, 2020

DOI: $10.5430 /$ crim.v7n3p11

URL: https://doi.org/10.5430/crim.v7n3p11

\begin{abstract}
$\mathrm{AL}$ amyloid cardiomyopathy is an increasingly recognized condition but arises less commonly with coexistent multiple myeloma. In this case report, we present a case of restrictive cardiomyopathy caused by AL amyloidosis and multiple myeloma, identified and treated using a multidisciplinary approach.
\end{abstract}

Key Words: Amyloidosis, Multiple myeloma, Restrictive cardiomyopathy, Heart failure

\section{History OF PRESENT ILlNESS}

A 54-year-old woman with a history of recent stroke, reported inferior myocardial infarction, type II diabetes, dyslipidemia, presented to the emergency department with worsening dyspnea on exertion and lower extremity edema. Four months before her presentation, she began to experience fatigue, exertional dyspnea, and lower extremity edema, and furosemide was prescribed. Two months prior to her presentation, she had an episode of expressive aphasia and right facial droop and was diagnosed with a left anterior cerebral artery stroke. Her symptoms resolved without treatment. Transthoracic echocardiogram at the time reported an ejection fraction of $35 \%-40 \%$ with septal hypokinesis and no cardiac source of embolism identified. Despite treatment with furosemide, she continued to have worsening shortness of breath, lower extremity edema, and developed 5-pillow orthopnea.

Her social history is notable for smoking until 5 months prior to presentation. Her father had an unspecified heart disease.

\section{INVESTIGATIONS}

A 12-lead electrocardiogram was obtained which showed diffuse low voltage, poor precordial $\mathrm{R}$ wave progression, and $\mathrm{q}$ waves in the anterior and inferior leads. Chest x-ray revealed mild cardiomegaly and NTproBNP was 7,503 pg/ml. She was admitted to cardiology and diuretics were initiated. Telemetry monitoring revealed sinus bradycardia followed by sinus node arrest with ventricular escape and isorhythmic atrioventricular dissociation (see Figure 1).

Echocardiogram revealed a non-dilated left ventricle (inferolateral [posterior] wall thickness of $1.6 \mathrm{~cm}$ ), LVEF 40\%-45\% (hypokinetic anterior and inferior wall segments), a restrictive filling pattern, and a $1.3 \mathrm{~cm} \times 1.0 \mathrm{~cm}$ round, highly mobile mass in the left atrium prolapsing into the mitral valve most consistent with a thrombus.

Given the electrocardiographic and echocardiographic abnormalities, cardiac amyloidosis was considered. Serum protein electrophoresis showed hypogammaglobulinemia without any monoclonal immunoglobulin. There was a highly abnormal serum light chain ratio (free kappa: 6.6, free lambda: 724.7, free kappa/lambda ratio: 0.01) and urine protein electrophoresis showed monoclonal free lambda light chains. PYP scan demonstrated mild cardiac pyrophosphate uptake. Cardiac magnetic resonance demonstrated diffuse early and

*Correspondence: Varayini Pankayatselvan; Email:vpankay1@bidmc.harvard.edu; Address: Beth Israel Deaconess Medical Center, United States. 
late gadolinium enhancement, consistent with cardiac amyloidosis. Interestingly, there was no evidence of nephrotic syndrome, neuropathy, hypercalcemia, renal dysfunction, anemia, or lytic bone lesions on skeletal survey or PET scan.

A hematology consultation was requested, and bone marrow biopsy revealed a $40 \%$ plasma cell population which was lambda restricted, with concurrent flow cytometry detecting a monoclonal lambda cell population; she was ultimately diagnosed with light chain multiple myeloma based upon a free light chain ratio of $<0.01$. Bone marrow and fat pad biopsies both stained positive for Congo red, and she was also diagnosed with AL amyloidosis.

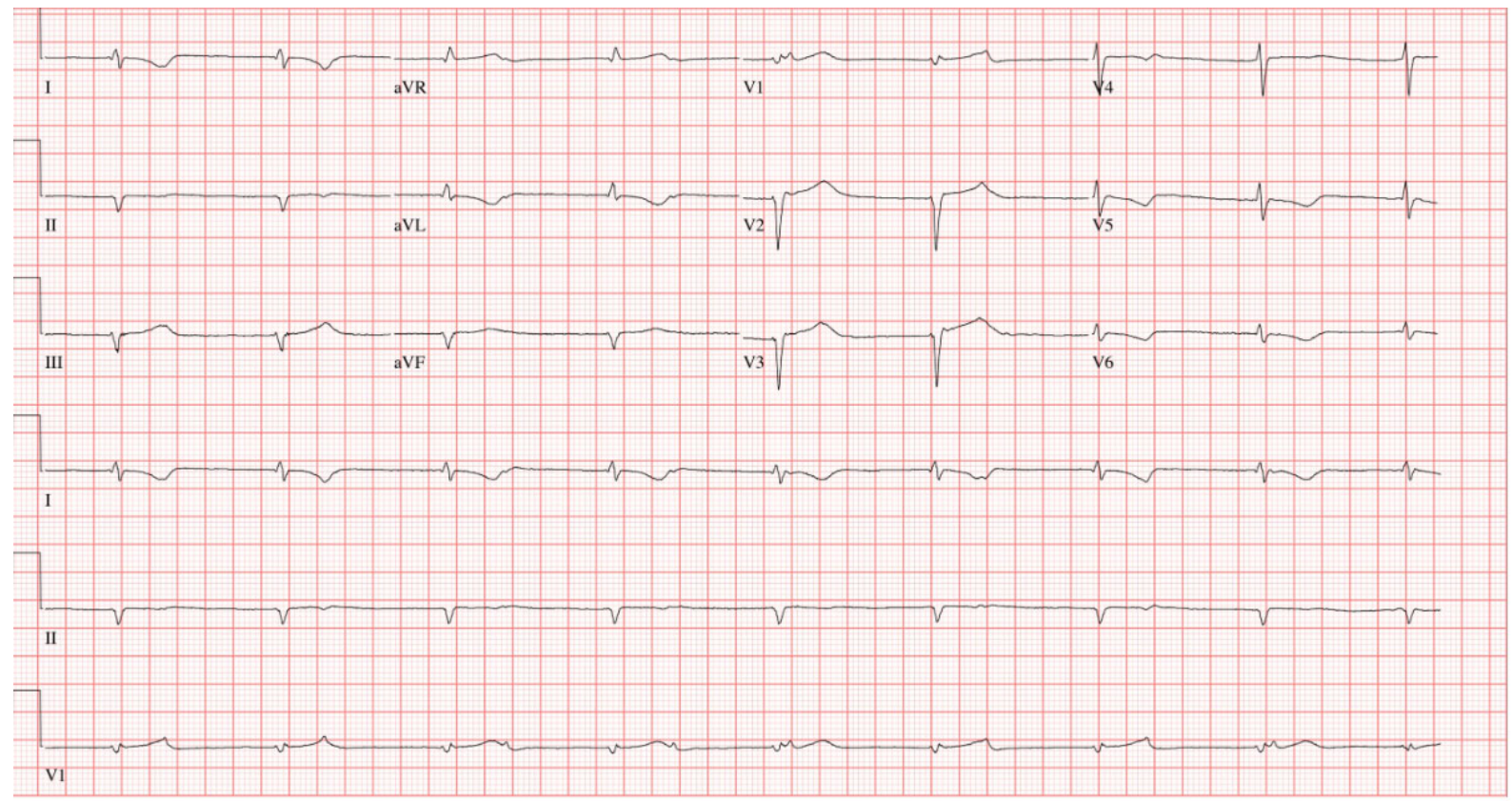

Figure 1. Isorhythmic atrioventricular dissociation

\section{Management}

She received intravenous diuresis but remained with signs of volume overload on physical exam in the setting of declining renal function, so she underwent right heart catheterization which demonstrated elevated filling pressures and a low cardiac index (right atrium $15 \mathrm{mmHg}$, pulmonary artery 42/22 $\mathrm{mmHg}$, pulmonary capillary wedge pressure $23 \mathrm{mmHg}$, cardiac output $1.89 \mathrm{~L} / \mathrm{min}$ and cardiac index $1.1 \mathrm{~L} / \mathrm{min} / \mathrm{m}^{2}$ ). Intravenous furosemide was increased and dopamine was initiated with notable improvement of dyspnea on exertion and a greater than 20-pound weight loss. Additionally, she was treated with intravenous heparin for the left atrial thrombus and planned for ongoing oral anticoagulation as outpatient. She was additionally monitored on telemetry during her 3 week hospitalization with no evidence of paroxysmal atrial fibrillation. For her multiple myeloma, she began chemotherapy with cyclophosphamide, bortezomib and dexamethasone (CyBorD) as recommended by hematology.

\section{FOLLOW-UP}

After the diagnoses of multiple myeloma and AL amyloidosis were made, she was evaluated for simultaneous bone marrow and cardiac transplant. Unfortunately, she was not a candidate for transplant and was discharged with plan to continue CyBorD for multiple myeloma and maximum tolerated heart failure therapy for the cardiac AL amyloidosis.

\section{DiscuSSION}

Amyloidosis is not a single disease entity but rather one in which over 20 proteins have been identified causing similar misfolding patterns resulting in tissue deposition. ${ }^{[1]}$ Three major systemic subtypes that specifically affect the heart are: ATTR-m (heritable), ATTR-wild type (senile), and AL amyloid. This discussion will focus primarily on the diagnosis and treatment of $\mathrm{AL}$ amyloidosis.

The clinical presentation of cardiac AL amyloidosis includes classic heart failure symptoms which are typically more rapid in onset compared to ATTR subtypes. Patients may also complain of angina, which is likely caused by microvascular amyloid deposition. Exam findings of orthostasis, macroglossia, periorbital bruising, and peripheral neuropathy should greatly increase suspicion for AL amyloidosis. ${ }^{[2]}$

The ECG findings in Figure 1 of low voltage, poor precordial 
$\mathrm{R}$ wave progression, and pseudo infarct patterns have been reported in other cases of AL cardiac amyloidosis. ${ }^{[3,4]}$ While low voltage is not always present, there is often a disconnect between voltage observed on ECG and the wall thickness observed on echocardiogram. Conduction disease is also classically seen in cardiac amyloidosis, the pathophysiology of which may partly be due to the infiltrative component of amyloid as well as its toxic effects on myocardial tissues. ${ }^{[5]}$

The presence of intracardiac thrombi on echocardiogram, most often occurring in the left atrial appendage, are more common in AL than TTR cardiac amyloidosis. ${ }^{[6]}$ Our patient presented with a recent stroke, likely caused from embolization of her left atrial thrombus, however continuous electrocardiographic monitoring was pursued to evaluate for paroxysmal atrial fibrillation. Anticoagulation may be considered in patients with evidence of atrial contractile dysfunction due to AL amyloidosis, even in the absence of atrial fibrillation. ${ }^{[6]}$

The diagnosis of amyloidosis is ultimately based on histology staining positive for Congo red. ${ }^{[7]}$ The abdominal fat pad is often biopsied due to ease and safety of procedure, though it can often have inadequate amyloid deposits to definitively subtype disease. Endomyocardial biopsy can also be performed, though is a more invasive diagnostic strategy. The type of amyloid is then determined by immunohistochemistry or mass spectrometry. If a monoclonal protein population exists, the subtype is typically AL, however it is critical to confirm AL, as a patient with concurrent ATTR amyloidosis and MGUS could be confused with having AL amyloid. ${ }^{[8]}$ It is important to note that AL amyloid can co-exist with multiple myeloma in about $5 \%-10 \%$ of patients, and portends a poor prognosis with one case series of $147 \mathrm{AL}$ amyloid patients showing a median survival of 14 months among patients with multiple myeloma compared to 33 months for those without multiple myeloma. ${ }^{[9]}$ Therefore bone marrow biopsy should also be performed for cases of suspected AL amyloid. $^{[10]}$
Treatment of AL cardiac amyloidosis involves chemotherapy and optimization of the patient's volume status. Cyclophosphamide, bortezomib and dexamethasone (CyBorD) has become a mainstay in treatment. ${ }^{[11]}$ Curative intent is possible with autologous bone marrow transplant for selected patients. Heart failure optimization is often challenging in patients with AL cardiac amyloidosis; neurohormonal antagonists such as beta blockers and ACE inhibitors are not well-tolerated, likely due to autonomic dysfunction and exaggerated restrictive pathophysiology. ${ }^{[10]}$ Left ventricular assist devices are rarely pursued given difficulty of restrictive physiology. Transplantation may be considered in select cases.

\section{Conclusion}

This case highlights the importance of an evaluation for infiltrative cardiomyopathies when similar ECG and echocardiography findings are encountered, as well as the importance of considering concurrent multiple myeloma in patients with AL amyloidosis.

\section{Highlights}

- To suspect amyloidosis in patients with a lower than expected ECG voltage compared to the observed ventricular wall thickness on echocardiogram

- To note that AL amyloid cardiomyopathy is an increasingly recognized condition but arises less commonly with coexistent multiple myeloma

- To consider anticoagulation in patients with cardiac amyloidosis and evidence of atrial contractile dysfunction, even in the absence of atrial fibrillation

- To understand definitive diagnosis of amyloidosis often requires endomyocardial biopsy to identify the subtype of protein deposits, which can dictate treatment

\section{CONFLicts OF InTEREST Disclosure}

The authors have declared no conflicts of interest.

\section{REFERENCES}

[1] Iadanza MG, Jackson MP, Hewitt EW, Ranson NA, Radford SE. A new era for understanding amyloid structures and disease. Nat Rev Mol Cell Biol. 2018; 19(12): 755-773. PMid:30237470. https : //doi.org/10.1038/s41580-018-0060-8

[2] Beel K, Vekemans M-C, Bries G, et al. Diagnosis and treatment of AL Amyloidosis in 2015: Consensus guidelines of the Belgian Hematological Society. 2015; 6(5): 8.

[3] Mussinelli R, Salinaro F, Alogna A, et al. Diagnostic and Prognostic Value of Low QRS Voltages in Cardiac AL Amyloidosis: Low QRS Voltages in Cardiac AL Amyloidosis. Ann Noninva- sive Electrocardiol. 2013; 18(3): 271-280. PMid:23714086. https : //doi.org/10.1111/anec. 12036

[4] Murtagh B, Hammill SC, Gertz MA, et al. Electrocardiographic findings in primary systemic amyloidosis and biopsy-proven cardiac involvement. Am J Cardiol. 2005; 95(4): 535-537. PMid:15695149. https : //doi.org/10.1016/j.amjcard.2004.10.028

[5] Liao R, Jain M, Teller P, et al. Infusion of Light Chains From Patients With Cardiac Amyloidosis Causes Diastolic Dysfunction in Isolated Mouse Hearts. Circulation. 2001; 104(14): 1594-1597. PMid:11581134. https://doi.org/10.1161/circ.104.14.15 94

Published by Sciedu Press 
[6] Feng D, Syed IS, Martinez M, et al. Intracardiac Thrombosis and Anticoagulation Therapy in Cardiac Amyloidosis. Circulation. 2009; 119(18): 2490-2497. PMid:19414641. https ://doi .org/10.116 1/CIRCULATIONAHA . 108.785014

[7] Comenzo RL, Reece D, Palladini G, et al. Consensus guidelines for the conduct and reporting of clinical trials in systemic light-chain amyloidosis. Leukemia. 2012; 26(11): 2317-2325. PMid:22475872. https://doi.org/10.1038/leu.2012.100

[8] Comenzo RL, Zhou P, Fleisher M, et al. Seeking confidence in the diagnosis of systemic AL (Ig light-chain) amyloidosis: patients can have both monoclonal gammopathies and hereditary amyloid proteins. Blood. 2006; 107(9): 3489-3491. PMid:16439680. https://doi.org/10.1182/blood-2005-10-4148
[9] Pardanani A, Thomas EW, Georgene S, et al. Circulating Peripheral Blood Plasma Cells as a Prognostic Indicator in Patients with Primary Systemic Amyloidosis. Blood 101, no. 3 (February 1, 2003): 827-30. PMid:12393530. https://doi.org/10.1182/blood-2 002-06-1698

[10] Falk RH, Alexander KM, Liao R, et al. AL (Light-Chain) Cardiac Amyloidosis: A Review of Diagnosis and Therapy. J Am Coll Cardiol. 2016; 68(12): 1323-1341. PMid:27634125. https : //doi.org/10.1016/j.jacc. 2016.06.053

[11] Jimenez-Zepeda VH, Duggan P, Neri P, et al. Bortezomib-Containing Regimens for the Treatment of Newly Diagnosed and Relapsed Amyloid Light Chain Amyloidosis: A Single-Center Experience. Clin Lymphoma Myeloma Leuk. 2016; 16(6): e79-e84. PMid:27101985. https://doi.org/10.1016/j.clml.2016.03.005 